\title{
Anabases
}

ANABASES Traditions et réceptions de l'Antiquité

$18 \mid 2013$

Varia

\section{John DAVIES, John WILKES éd., Epigraphy and the} historical sciences

\section{Monique Dondin-Payre}

\section{(2) OpenEdition}

\section{Journals}

Édition électronique

URL : http://journals.openedition.org/anabases/4393

DOI : 10.4000/anabases.4393

ISSN : 2256-9421

\section{Éditeur}

E.R.A.S.M.E.

\section{Édition imprimée}

Date de publication : 1 octobre 2013

Pagination : 274-276

ISSN : 1774-4296

\section{Référence électronique}

Monique Dondin-Payre, "John DAvies, John wiLkes éd., Epigraphy and the historical sciences », Anabases [En ligne], 18 | 2013, mis en ligne le 01 janvier 2013, consulté le 22 septembre 2020. URL : http:// journals.openedition.org/anabases/4393; DOI : https://doi.org/10.4000/anabases.4393

Ce document a été généré automatiquement le 22 septembre 2020.

(c) Anabases 


\title{
John DAVIES, John WILKES éd., Epigraphy and the historical sciences
}

\author{
Monique Dondin-Payre
}

\section{RÉFÉRENCE}

John DAVIES, John wILKES éd., Epigraphy and the historical sciences, Oxford, Oxford

University Press, Proceedings of the British Academy 177, 2012, 346 p.

75 livres / ISBN 978-0-19-726506-2.

1 Ce volume rassemble la plupart des conférences plénières données lors $d u 13^{\mathrm{e}}$ congrès international d'épigraphie grecque et latine (CIEGL) qui s'est tenu à Oxford en septembre 2007. Il avait été demandé aux auteurs de mettre en évidence les apports de l'épigraphie à l'écriture de l'histoire, ce qui fait apparaître combien, à l'inverse, pour exploiter les inscriptions, c'est-à-dire pour en tirer les informations, les insérer dans un contexte et apprécier leurs apports, il est nécessaire de maîtriser l'histoire: le déchiffrement d'une inscription n'est que la première étape d'une analyse historique. Les contributeurs ont donc été invités à axer leurs propos sur la place de l'épigraphie dans des domaines historiques spécifiques. Le contrat est pleinement rempli en ce qui concerne la religion, parce que Robert Parker (p. 17-30) pour la période grecque, John Scheid (p. 31-44) pour la période romaine, déterminent les types de documents qui contribuent à la connaissance de pratiques et de rites qui relèvent pourtant de la transmission orale. Ces catégories sont similaires pour les deux époques: listes de prêtres, tablettes d'exécration, lois sacrées, calendriers, dédicaces... sont nombreux mais disparates car leur propos n'est pas d'exposer un rituel enregistré dans la mémoire collective mais d'en énoncer des exceptions, des innovations, des réactions à des imprévus. J. Scheid insiste sur le fait que, les inscriptions faisant partie du rituel dans la religion romaine, elles sont codifiées et ne tiennent donc pas compte des incidents, des accidents, des omissions : par rapport à la littérature, les inscriptions ne décrivent pas le déroulement concret des rites, elles attestent que les rapports avec la 
divinité ont été gérés selon les règles. Pour le reste, le volume est maladroitement construit: la logique de l'agencement des parties est insolite et, surtout, l'équilibre entre les langues n'est pas du tout respecté. Le grec est surreprésenté par rapport au latin, une forte proportion d'études s'inscrivant dans la chronologie romaine se fixe sur l'épigraphie en grec. Il en va ainsi pour l'économie, l'autre domaine où époques grecque et romaine sont envisagées parallèlement. Tant Alain Bresson (p. 223-247) que Giovanni Salmeri (p. 249-267) dessinent l'évolution qui a substitué à l'approche anthropologique (Finley, Rostovtzeff) un point de vue rationaliste qui prend en compte les contraintes institutionnelles, d'où l'élaboration de nouveaux concepts. Le propos des inscriptions n'est pas d'exposer des faits économiques, elles fournissent donc des informations parcellaires et immédiates qui doivent être organisées pour dégager des mouvements de longue durée. Mais l'épigraphie permet d'aborder des questions concrètes telles que les régimes d'exploitation agricole, les prix, les impôts... Les deux contributions sont très similaires, Salmeri examinant les locuteurs grecs d'époque romaine; signalons l'originalité de l'analyse des inscriptions sur les tuiles des mines de sulfure siciliennes développée par ce dernier. Bien qu'elles soient regroupées sous l'intitulé «Structures de pouvoir ", les contributions de John Ma (p. 133-158) et d'Alison Cooley (p. 159-182) ne permettent pas de confronter les pratiques grecques et romaines en ce domaine, une comparaison pourtant édifiante étant donné le gouffre qui sépare les systèmes politiques des cités autonomes grecques de l'empire romain. J. Ma prend des exemples grecs de toutes les époques et les met en relation avec des parallèles contemporains, pour démontrer de façon convaincante et vivante le fait que, indépendamment de la source de l'inscription, l'affichage fonctionne comme si le texte exposé aux yeux de tous avait une efficacité en lui-même, le choix de son emplacement étant crucial pour qu'il ait tout son pouvoir. A. Cooley élabore un panorama de la diffusion des documents émis à Rome dans la partie orientale de l'empire, en insistant sur les modalités variées de cette exposition publique, la pierre et le bronze étant moins usuels que les inscriptions éphémères sur bois, et sur l'initiative des autorités locales, collectives ou individuelles, dans les décisions d'affichage, qui, les associant au pouvoir central, les mettent elles-mêmes en valeur. En dépit du titre commun "Épigraphie et population ", les développements sur la connaissance des populations (Christof Schuler, p. 63-100) et sur la démographie (Walter Scheidel, p. 101-129) n'ont guère en commun que le support linguistique grec. Le premier mène une analyse approfondie, mais circonscrite aux communautés villageoises d'Asie, qui, nombreuses et vivantes, développent les moyens d'exprimer leurs préoccupations, différentes de celles des villes, sur les récoltes, le temps, le bétail, les cultures. Bien menée mais très pointue, cette réflexion n'appartient pas au registre des synthèses, alors que les apports et limites de l'épigraphie dans le domaine démographique font l'objet d'un développement méthodologique général bien structuré qui insiste justement sur la vigilance extrême que l'imprécision des âges rend indispensable. Les apports de l'épigraphie à la connaissance des langues ne sont envisagés que pour le grec (Georg Petzl, p.47-60), alors que l'empire romain et l'uniformisation linguistique, ses modalités, ses limites, ses réussites auraient constitué un terrain beaucoup plus propice à ces considérations. L'onomastique, un des champs dans lesquels l'épigraphie est le plus fondamentale, n'est traitée que dans l'introduction, par Denis Feissel (p.1-14), qui concentre les exemples de son exposé général sur l'onomastique byzantine en langue grecque. Enfin, on comprend difficilement pourquoi les considérations fouillées et réalistes d'Isabel Rodà (p. 185-219) sur la meilleure façon de présenter les inscriptions dans les musées pour que le public 
les apprécie et les comprenne, et celles de Silvio Panciera et John Bodel (p. 271-296) sur l'apport fondamental d'internet (corpus en évolution permanente, images, interrogations des bases...), sont séparées par le chapitre sur l'économie. Angelos Chaniotis (p. 299-328) met heureusement en évidence que, puisque saxa loquuntur, il convient d'écouter les émotions, les reflets d'humanité qu'émettent les acclamations, les lamentations, les prières..., une conclusion plaisante et originale à un volume déconcertant par sa structure peu cohérente, qui passe du singulier au général, de la synthèse pour étudiants ou débutants aux développements pointus, dans lequel on ne trouvera ni bilan des apports de l'épigraphie dans les différents registres historiques ni réflexion synthétique sur les relations entre l'épigraphie et l'histoire. Un index et une liste des sources permettront l'exploitation optimale de cette publication qui mérite de retenir l'attention, mais reste trop disparate.

\section{AUTEURS}

\section{MONIQUE DONDIN-PAYRE}

CNRS - Paris, UMR 8210

dondin_payre@club-internet.fr 